\title{
RESEARCH PAPER \\ AEROBIC BACTERIA IN SAFE TYPE CHRONIC SUPPURATIVE OTITIS MEDIA IN GEZIRA STATE, SUDAN
}

\author{
M. EImustafa ${ }^{1}$, M. A. Yousif ${ }^{2}$, W. N. Osman ${ }^{3}$ and O. M. Elmustafa ${ }^{4}$ \\ ${ }^{1}$ Faculty of Pharmacy, University of Gezira, Sudan \\ Email: elmustafa@uofg.edu.sd \\ ${ }^{2}$ College of Pharmacy, Taif University, Saudi Arabia \\ Email:mirghani53@yahoo.com \\ ${ }^{3}$ Faculty of Medicine, University of Gezira, Sudan \\ Email:wailnuri2000@yahoo.co.uk \\ ${ }^{4}$ Faculty of Medicine, Wad Medani College for Medical Sciences and Technology, Sudan \\ Email:abshama50@yahoo.com
}

\begin{abstract}
Chronic suppurative otitis media (CSOM) is a challenging disease with a high burden on society in developing countries. Information regarding CSOM in Sudanese patients is scarce. This study aimed to identify aerobic bacteria involved in CSOM and to determine their sensitivity towards commonly prescribed antibiotics. A multi-center prospective cross sectional study was conducted between June 2012 and October 2013 in private and public ENT clinics in Gezira State, Sudan. The study included 217 CSOM patients most of whom were males or had unilateral disease. The most common isolates were Staphylococcus aureus (42.6\%), Klebsiella pneumoniae (14.7\%), Pseudomonas aeroginosa (12.5\%) and Proteus mirabilis (10.2\%). The sensitivity of all isolates was highest towards gentamicin and ciprofloxacin while multiple drug resistance was prominent towards amoxyclav, cotrimoxazole, cefuroxime, cefaclor and ceftriaxone. Ciprofloxacin and gentamicin appear to be the best choices for empiric therapy in patients with CSOM. In contrast, the use of amoxyclav, cotrimoxazole, cefuroxime, cefaclor and ceftriaxone should be discouraged.
\end{abstract}

Keywords: Aerobic bacteria, Chronic suppurative otitis media, Gezira State, Sudan

\section{INTRODUCTION}

Chronic suppurative otitis media (CSOM) is a persistent inflammatory disorder of the middle ear and mastoid cavity. It is characterized by discharge into the external ear canal over two to six weeks through a perforation of the tympanic membrane (Aduda et al, 2013; Acuin, 2004). Worldwide changes in the resistance to antim- icrobials and distribution of organisms causing CSOM have been detected (Lee et al, 2014; Myer et al, 2013). In the Gezira State of Sudan, patients are usually seen by an Otorhinolaryngologist after multiple visits to primary health care centres and in many cases after venturing with antibiotics obtained over the counter from local pharmacies. Antimicrobial treatment is 
usually chosen on an empirical basis with no preceding culture or sensitivity testing.

This study aimed to identify pathogenic aerobic bacteria in CSOM patients in the Gezira State of Sudan and to determine their sensitivity towards commonly prescribed antibiotics.

\section{METHODS}

This prospective, cross sectional multicenter study was conducted at the Ear, Nose and Throat departments of Wad Medani Teaching Hospital and Almanagil Hospital as well as at private ENT clinics in both cities between June 2012 and October 2013. These facilities are the only ones that provide ENT services on a regular basis to a population of $3,780,915$ in the Gezira State (Central Bureau of Statistics, 2008).

Patients were included if otoscopy revealed a centrally perforated ear drum with visible non offensive mucopurulent discharge in the external ear canal for at least two weeks prior to presentation. They were excluded if they had symptoms for less than two weeks, took antimicrobial treatment during the past two weeks, in case of presence of otitis externa, otomycosis or cholesteatoma.

After cleansing of the external ear, an ENT consultant or registrar performed bilateral otoscopy and collected a single specimen from eligible ears using sterile fine cotton swabs. Swabs were inoculated onto blood agar, chocolate agar and MacConkey agar and incubated for up to 48 hours at $35-37^{\circ} \mathrm{C}$. Isolates were identified using standard laboratory techniques including microscopy, gram staining and biochemical testing. Antimicrobial susceptibility was determined using the modified Kirby Bauer disc diffusion test (Cheesbrough, 2006) and interpreted using Clinical and Laboratory Standards Institute (CLSI) guidelines (CLSI, 2007).

The study was approved by the University of Gezira Ethical Review Committee.

\section{RESULTS}

Two hundred and seventeen patients met the inclusion criteria with a median age of 20 years. Ninety patients $(41.7 \%)$ were in the age group less than 16 years, 50 patients $(23.1 \%)$ were aged 16-30 years, 37 patients $(17.1 \%)$ were $31-45$ years old, 31 patients $(14.4 \%)$ were in the age group 46-60 years and 8 patients $(3.7 \%)$ were older than 60 years of age. Of these patients, $83(38.2 \%)$ were seen at a hospital settings while the remaining $134(61.8 \%)$ were seen at private clinics. The male to female ratio was $1: 1.4$ and most patients $(n=160$, $73.7 \%$ ) resided in rural areas. Patients with bilateral disease were $55(25.3 \%)$, which was less than those with unilateral disease numbering $162(74.4 \%)$ and the ratio was 1:2.9.

From the 217 included patients, 40\% $(n=87)$ received oral antibiotics, $11 \%(\mathrm{n}=24)$ received topical antibiotics and $49 \%(n=106)$ received a combination of both. The antibiotic prescription frequency was 323 antibiotics for all patients giving an average of 1.5 antibiotics per patient encounter. Ninety four incidents $(29.1 \%)$ were prescribed using the generic name while the remaining $229(70.9 \%)$ were prescribed using the brand name.

In total 272 ear swabs were collected from the included patients. About $87 \%(n=236)$ of swabs were culture positive and $13 \%$ had no microbial growth. $78 \%$ of the cultures contained single organisms while poly-microbial growth occurred in $22 \%$ of cultures. The most common isolates were Staphylococcus aureus (42.6\%), Klebsiella pneumoniae (14.7\%), Pseudomonas aeroginosa $(12.5 \%)$ and Proteus mirabilis $(10.2 \%)$. The remaining isolates included Staphylococcus epidermidis, Staphylococcus saprophyticus, Streptococcus spp., Proteus vulgaris and Escherichia coli all of which constituted $20 \%$ of the isolates.

While most isolates remained fairly sensitive towards Ciprofloxacin and Gentamicin, the resistance was highest towards Cotrimoxazole, Amoxyclav, Cefuroxime, Cefaclor and Ceftri- 
axone. All microorganisms showed higher sensitivity towards Gentamicin compared to Ciprofloxacin except for Proteus mirabilis (Table 1).

For Staphylococcus aureus, 93\% of isolates showed resistance to more than one antibiotic. Of these strains, $47 \%$ were resistant to at least five of the tested antibiotics. Similarly, Pseudomonas aeroginosa isolates showed marked multi drug resistance with all organisms being resistant to at least three of the test antibiotics. Also multi drug resistance was very apparent with Proteus mirabilis and Klebsiella pneumoniae isolates (Table 2 and 3 ).

\section{DISCUSSION}

Knowing prevalent bacterial patterns in the community is crucial for effective planning of treatment. The most common isolates identified in this study were $S$. aureus, $K$. pneumoniae, $P$. aeroginosa and $P$. mirabilis. In similar studies, $S$. aureus and $P$. aeroginosa were the most commonly identified bacteria (Aduda et al, 2013; Myer et al, 2013; Vishwanath et al, 2012; Cheong et al, 2012; and Brook, 2008). The detection of K. pneumoniae and P. Mirabilis has also become common in developing countries (Aduda et al, 2013; Myer et al, 2013; Seid et al, 2013, and Lodhi et al, 2010). This relatively

high prevalence of Proteus and Klebsiella spe- cies indicates poor environmental hygiene and sanitation patterns in our community. Thus, appropriate public health interventions alongside clinical management are critical in combating both bacterial resistance and CSOM recurrence.

While most bacteria remained fairly sensitive towards Ciprofloxacin and Gentamicin, resistance was highest towards Cotrimoxazole, Amoxyclav, Cefuroxime, Cefaclor and Ceftriaxone. These are the most widely prescribed and probably most widely misused antibiotics in our communities. The marked resistance is evidence beyond doubt to the extent of irrational antibiotic prescribing in Sudan. No other study has indicated such levels of marked resistance among the different species of pathogenic agents in CSOM towards so many antibiotics. In addition, the level of multidrug resistance revealed in this study was remarkably high. About $78 \%$ of our isolated bacteria were resistant to at least four antibiotics. This figure exceeds that described by Alsaimary et al (2010) who described high multidrug resistance in $P$. aeroginosa and $S$. aureus and similar to that described by Seid et al (2013).

Large variations in resistance towards one class of antibiotic or the other between geographical

Table 1: Antibiotic Sensitivity Pattern of Isolates from CSOM Patients

\begin{tabular}{|c|c|c|c|c|c|c|}
\hline & $\begin{array}{l}\text { S. aureus } \\
(N==130) \\
n(\%)\end{array}$ & $\begin{array}{l}\text { K. pneumo- } \\
\text { niae }(N=45) \\
n(\%)\end{array}$ & $\begin{array}{l}P . \text { aerogi- } \\
\text { nosa }(N=38) \\
n(\%)\end{array}$ & $\begin{array}{l}\text { P. mirabi- } \\
\text { lis }(N=31) \\
n(\%)\end{array}$ & $\begin{array}{l}\text { Other } \\
\text { organisms } \\
(N=61) \\
n(\%)\end{array}$ & $\begin{array}{l}\text { All isolates } \\
(N=305) \\
n(\%)\end{array}$ \\
\hline Ciprofloxacin & $95(73 \%)$ & $37(82 \%)$ & $24(63 \%)$ & $28(90 \%)$ & $52(85 \%)$ & $236(77 \%)$ \\
\hline Gentamicin & $112(86 \%)$ & $39(87 \%)$ & $24(63 \%)$ & $26(84 \%)$ & $52(85 \%)$ & $253(83 \%)$ \\
\hline Cotrimoxazole & $61(47 \%)$ & $27(60 \%)$ & $8(21 \%)$ & $13(42 \%)$ & $0(0 \%)$ & $109(36 \%)$ \\
\hline Amoxyclav & $58(45 \%)$ & $15(33 \%)$ & $10(26 \%)$ & $12(39 \%)$ & $0(0 \%)$ & $95(31 \%)$ \\
\hline Cefuroxime & $61(47 \%)$ & $15(33 \%)$ & $8(21 \%)$ & $14(45 \%)$ & $0(0 \%)$ & $98(32 \%)$ \\
\hline Cefaclor & $61(47 \%)$ & $14(31 \%)$ & $0(0 \%)$ & $14(45 \%)$ & $0(0 \%)$ & $89(29 \%)$ \\
\hline Ceftriaxone & $77(59 \%)$ & $14(31 \%)$ & $8(21 \%)$ & $15(48 \%)$ & $0(0 \%)$ & $114(37 \%)$ \\
\hline
\end{tabular}



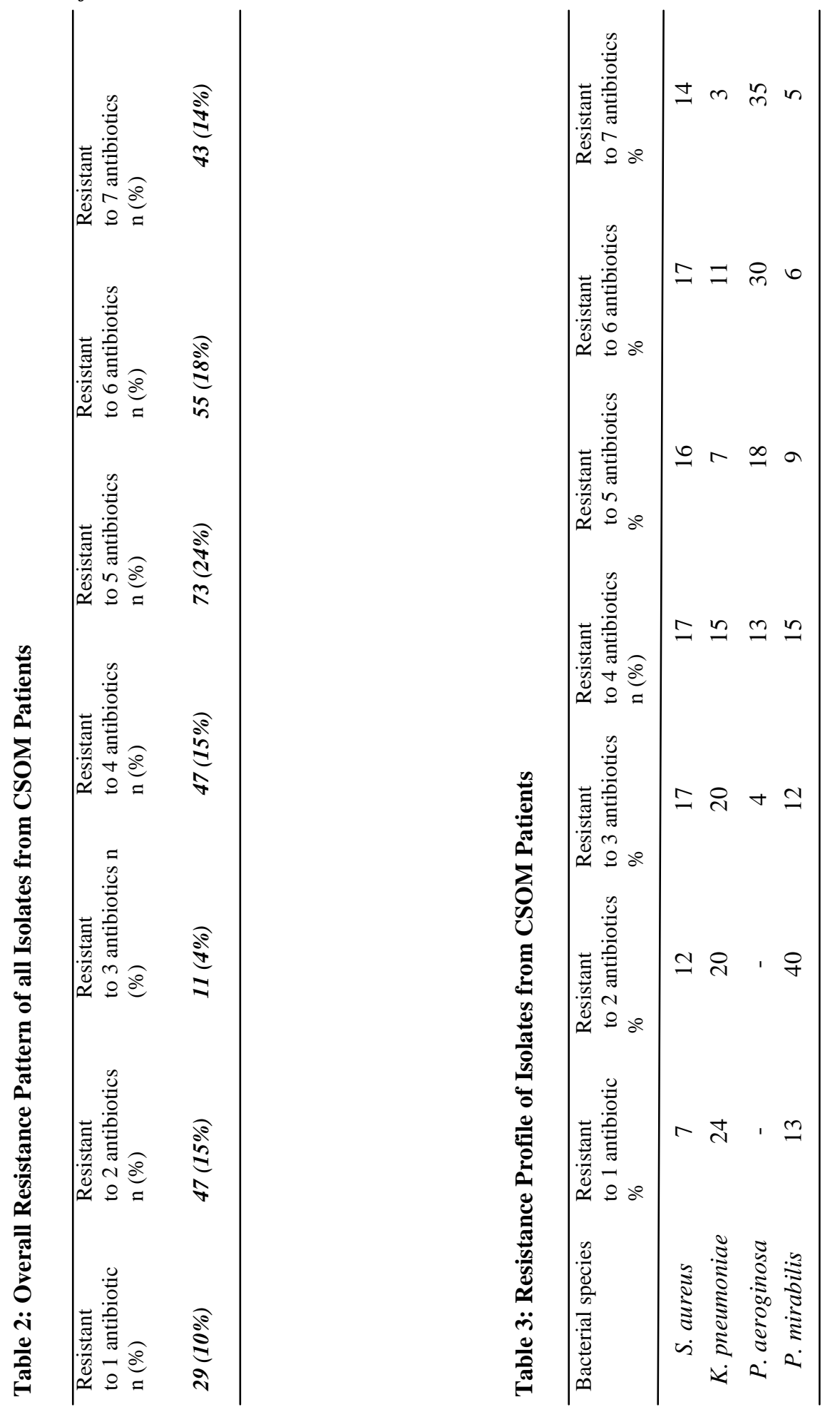

Journal of Science and Technology @ KNUST April 2016 
regions points a suspicious finger towards management practices as a major force behind this resistance. This is clear as higher levels of resistance are almost always associated with the most prescribed antibiotics as in the current case or as reported by other study groups (Cheong et al, 2012; Alsaimary et al, 2010).

\section{CONCLUSION}

Using oral antibiotics alone or in combination with topical ones is very common in managing CSOM. Staphylococci particularly, Staphylococcus aureus in addition to Klebsiella pneumoniae, Proteus mirabilis and Pseudomonas aeroginosa constitute almost $90 \%$ of aerobic bacteria associated with CSOM. Most bacterial isolates from CSOM patients in the Gezira State have demonstrated multi drug resistance. More than $78 \%$ of isolates were resistant to at least four commonly used antibiotics. Bacterial isolates in CSOM patients are most sensitive towards Gentamicin and Ciprofloxacin, but extensively resistant towards Amoxyclav, Cefuroxime, Cefaclor, Cotrimoxazole and Ceftriaxone.

\section{REFERENCES}

Acuin, J. (2004). Chronic suppurative otitis media: Burden of illness and management options. World Health Organization. Geneva, Switzerland.

Aduda, D. S. O., Macharia, I. M., Mugwe, P., Oburra, H., Farragher, B., Brabin, B. and Mackenzie, I. (2013). Bacteriology of chronic suppurative otitis media (CSOM) in children in Garissa district Kenya: A point prevalence study. International Journal of Pediatric Otorhinolaryngology, 77: 11071111.

Alsaimary, I. E., Alabbasi, A. M. and Najim, J. M. (2010). Impact of multi drugs resistant bacteria on the pathogenesis of chronic suppurative otitis media. African Journal of $M i$ crobiology Research, 13: 1373-1382.

Brook, I. (2008). Microbiology and Antimicro- bial Management of Head and Neck Infections in Children. Advances in Pediatrics, 55: 305-325.

Central Bureau of Statistics, (2008). The total population expected to States for the period 2009-2018. [Online] Available at: http:// www.cbs.gov.sd/en/files.php?id=7\#\&panel11 Accessed on 31 October 2015.

Cheesbrough, M. (2006). District Laboratory Practice in Tropical Countries Part 2. Cambridge University Press, UK.

Cheong, C., Tan, L. and Ngo, R. (2012). Clinical audit of the microbiology of otorrhoea referred to a tertiary hospital in Singapore, Singapore Medical Journal, 53(4): 244-248.

Clinical Laboratory Standards Institute (CLSI), (2007). Performance standards for antimicrobial susceptibility testing: 17th informational supplement. CLSI M100-S17;27(1). Wayne, PA, Accessed on $13^{\text {th }}$ November, 2013 fromhttp://www.microbiolab-bg.com/ CLSI.pdf

Lee, J. S., Kim, M. G., Hong, S. M., Na, S.Y., Byun, J. Y., Park, M. S. and Ye, S. G. (2014). Changing patterns of bacterial strains in adults and children with otitis media in Korean tertiary care centres. Clinical and Experimental Otorhinolaryngology, 7(2):7986.

Lodhi, M., Munir, T., Aziz, K. and Lodhi, H. (2010). Chronic suppurative otitis media; empiric quinolones in children. Professional Medical Journal, 17(3): 420-424.

Myer, E., Whitelaw, A., Edkins, O. And Fagan, J. J.(2013). Chronic otorrhoea: spectrum of microorganisms and antibiotic sensitivity in a South African cohort. South African Medical Journal, 103(7): 471-473.

Seid, A., Deribe, F., Ali, K. and Kibru, G. (2013). Bacterial otitis media in all age group 
of patients seen at Dessie referral hospital, North East Ethiopia. Egyptian Journal of Ear, Nose, Throat and Allied Sciences, 14:73 -78 .

Vishwanath, S., Mukhopadhyay, C., Prakash,
R., Pillai, S., Pujary, K. and Pujary, P. (2012). Chronic Suppurative Otitis Media: Optimizing Initial Antibiotic Therapy in a Tertiary Care Setup. Indian Journal of Otolaryngology Head and Neck Surgery, 64 (3):285-289. 